\title{
If the primes are finite, then all of them divide the number one
}

We propose a novel proof of the infinitude of the primes based on elementary considerations of Legendre's function $\phi$, defined in [1, p. 153] as

$$
\phi(x, y)=\mid\{1 \leq n \leq x \text { : integer } n \text { has no prime factors } \leq y\} \mid,
$$

where $x$ and $y$ are positive integers. The reader can see that

$$
\pi(x)=\pi(\sqrt{x})+\phi(x, \sqrt{x})-1,
$$

where $\pi(\cdot)$ is the prime-counting function. Let $p_{1}, \ldots, p_{s}$ be the prime numbers less than or equal to $y$. Using the inclusion-exclusion principle, it can be proved that

$$
\phi(x, y)=x-\sum_{1 \leq i \leq s}\left[\frac{x}{p_{i}}\right]+\sum_{1 \leq i, j \leq s}\left[\frac{x}{p_{i} p_{j}}\right]+\ldots+(-1)^{s}\left[\frac{x}{p_{1} \cdots p_{s}}\right],
$$

where [.] is the floor function. Pinasco [2] also used this principle for proving the infinitude of the primes, but his proof is remarkably different from ours.

Suppose that $\left\{p_{1}, \ldots, p_{s}\right\}$ is the set of all prime numbers. Consider $N=$ $p_{1} \cdots p_{s}$. Then $\phi\left(N^{2}, N\right)=1$. On the other hand, we have

$$
\phi\left(N^{2}, N\right)=N^{2}-\sum_{1 \leq i \leq s}\left[\frac{N^{2}}{p_{i}}\right]+\sum_{1 \leq i, j \leq s}\left[\frac{N^{2}}{p_{i} p_{j}}\right]+\ldots+(-1)^{s} N .
$$

Hence, $\phi\left(N^{2}, N\right)=m N$ for some integer $m$, i.e., every prime number divides 1 . This means that all primes, and, consequently, all non-zero natural numbers, are invertible in $\mathbb{N}$, i.e., we find that $\mathbb{N}$ is a field. This completes the proof.

\section{References}

[1] R. Crandall, C. Pomerance, Prime numbers. A computational perspective, Springer-Verlag, New York, 2nd Edition, 2005.

[2] J. P. Pinasco, New proofs of Euclid's and Euler's theorems, Amer. Math. Monthly 116 (2009) 172-174.

— Submitted by Umberto Cerruti and Nadir Murru 